\title{
Axiomatic derivation of the Doppler factor and related relativistic laws
}

\author{
Jean-Claude Falmagne and Jean-Paul Doignon
}

This paper is fondly dedicated to Janos Aczél, the first author's friend and mentor for more that four decades

\begin{abstract}
In the context of the relativistic Doppler effect [DE] and the Lorentz-Fitzgerald contraction $[\mathrm{LF}]$, we investigate the consequences of two abstract axioms $[R]$ and $[\mathrm{M}]$ expressed in terms of an operation $\oplus$ generalizing the addition of velocities and a function $L:(\lambda, v) \mapsto L(\lambda, v)$. The latter can represent either the Doppler effect or the LorentzFitzgerald Contraction. In words, these axioms state the following: $[\mathrm{R}]$ iterating the function $L$ has the same effect as adding velocities; $[\mathrm{M}]$ adding a velocity via the operation $\oplus$ preserves the order of the function $L$. We prove that these axioms are equivalent to each other, and also to generalized forms of the Doppler effect and the standard expression $[\mathrm{AV}]$ for the relativistic addition of velocities, taken jointly. We also show that if [AV] holds, then the axioms $[\mathrm{R}]$ and $[\mathrm{M}]$ are inconsistent with $[\mathrm{LF}]$.
\end{abstract}

Mathematics Subject Classification (2000). 65Q20, 83A01, 70A05.

Keywords. Axiomatics, Doppler effect, Lorentz-Fitzgerald Contraction, functional equations.

\section{Introduction}

Functional equations techniques may enable the gathering of diverse empirical phenomena under a single abstract axiomatic umbrella. We give an example here.

A relativistic Doppler effect arises when a source of light with wavelength $\lambda$ and an observer of that source are in relative motion with respect to each other. When the source and the observer are moving towards each other at a

We are grateful to Janos Aczél, Chris Doble, Michael Kiessling, Alexey Krioukov, David Malament, Pat Suppes, and a referee for their reactions to earlier versions of the results presented here. The second author's work is partially supported by an ARC grant from the Communauté Française de Belgique. 
speed $v$, the perceived wavelength $L(\lambda, v)$ increases in $\lambda$ and decreases in $v$, according to the special relativity formula

$$
[\mathrm{DE}] \quad L(\lambda, v)=\lambda \sqrt{\frac{1-\frac{v}{c}}{1+\frac{v}{c}}} \quad(\lambda>0,0 \leq v<c),
$$

in which: $c$ is the speed of light, $\lambda$ is the wavelength of the light emitted by the source, and $L(\lambda, v)$ is the wavelength of that light measured by the observer (cf. $[5,7])$.

This paper analyzes the relativistic Doppler effect [DE] in the context of two natural invariance axioms constraining the function $L$. These axioms are expressed in terms of an abstract operation

$$
\oplus:[0, c[\times[0, c[\rightarrow[0, c[
$$

representing the addition of velocities. Accordingly, we assume that $\oplus$ is continuous, strictly increasing in both variables.

These two axioms are as follows:

[R] $L(L(\lambda, v), w)=L(\lambda, v \oplus w) \quad(\lambda>0$, and $v, w \in[0, c[)$.

In words: iterating $L$ has the same effect as adding velocities via the operation $\oplus$.

[M] $L(\lambda, v) \leq L\left(\lambda^{\prime}, v^{\prime}\right) \Longleftrightarrow L(\lambda, v \oplus w) \leq L\left(\lambda^{\prime}, v^{\prime} \oplus w\right)\left(\lambda>0\right.$, and $v, v^{\prime}, w \in$ $[0, c[)$.

In words: adding a velocity via the operation $\oplus$ preserves the order of the function $L$.

If we assume that the operation $\oplus$ is the standard representation for the relativistic addition of velocities, that is,

$$
[\mathrm{AV}] \quad v \oplus w=\frac{v+w}{1+\frac{v w}{c^{2}}}
$$

these axioms are easy to interpret and intuitively cogent, at least a priori. However, we shall see that they have curious consequences.

The Lorentz-Fitzgerald Contraction will also be relevant to our discussion. It is expressed by the equation

$$
[\mathrm{LF}] \quad L(\lambda, v)=\lambda \sqrt{1-\left(\frac{v}{c}\right)^{2}} \quad(\lambda>0,0 \leq v<c),
$$

which describes the apparent decrease of the length of a rod, for an observer traveling on a line parallel to the rod. Thus, $\lambda$ is the length of the rod measured by an observer at rest with respect to the rod, and $L(\lambda, v)$ is the length of the same rod, as measured by an observer traveling at the speed $v$ with respect to the rod. For convenience, we use the same letter $L$ for the function in the Doppler effect and in the Lorentz-Fitzgerald Contraction, even though two different phenomena are at play. The reason for this choice of notation will soon be apparent. 
We will derive three sets of equivalences. In our first main result in Theorem 6 , we do not assume that the operation $\oplus$ has the form $[\mathrm{AV}]$. Under natural background conditions on the function $L$ (continuity, monotonicity), we prove that $[R]$ and $[M]$ are equivalent, and that these axioms are equivalent to the following generalizations of $[\mathrm{DE}]$ and $[\mathrm{AV}]$, taken jointly:

$$
\begin{aligned}
& {\left[\mathrm{DE}^{\dagger}\right] \quad L(\lambda, v)=\lambda\left(\frac{c-u(v)}{c+u(v)}\right)^{\xi},} \\
& {\left[\mathrm{AV}^{\dagger}\right] \quad v \oplus w=u^{-1}\left(\frac{u(v)+u(w)}{1+\frac{u(v) u(w)}{c^{2}}}\right) .}
\end{aligned}
$$

The letter $\xi$ denotes an arbitrary positive constant and $u$ is a continuous strictly increasing function mapping the interval $\left[0, c\left[\right.\right.$ onto itself. Thus, $\left[\mathrm{DE}^{\dagger}\right]$ and $\left[\mathrm{AV}^{\dagger}\right]$ generalize $[\mathrm{DE}]$ and $[\mathrm{AV}]$, which is obtained when $u$ is the identity function and $\xi=\frac{1}{2}$. Specifically, our Theorem 6 establishes the equivalences

$$
[\mathrm{R}] \Longleftrightarrow\left(\left[\mathrm{DE}^{\dagger}\right] \&\left[\mathrm{AV}^{\dagger}\right]\right) \Longleftrightarrow[\mathrm{M}] \text {. }
$$

Theorem 6 has two corollaries. In Corollary 7, we assume that the operation $\oplus$ satisfies the standard formula $[\mathrm{AV}]$ for the relativistic addition of velocities and we derive the stronger result:

$$
[\mathrm{AV}] \Longrightarrow\left([\mathrm{R}] \Longleftrightarrow\left[\mathrm{DE}^{\star}\right] \Longleftrightarrow[\mathrm{M}]\right) \text {, }
$$

with, for some positive constant $\xi$,

$$
\left[\mathrm{DE}^{\star}\right] \quad L(\lambda, v)=\lambda\left(\frac{1-\frac{v}{c}}{1+\frac{v}{c}}\right)^{\xi} \text {. }
$$

So, assuming that $[\mathrm{AV}]$ holds, each of the axioms implies the relativistic Doppler effect up to some positive exponent. However, this also implies that Axioms $[\mathrm{R}]$ and $[\mathrm{M}]$ are inconsistent with the Lorentz-Fitzgerald Contraction Formula $[\mathrm{LF}]$ if $\oplus$ satisfies $[\mathrm{AV}]$.

This inconsistency motivates Corollary 9, in which we do not assume that $\oplus$ satisfies $[\mathrm{AV}]$. Instead, we assume that $[\mathrm{LF}]$ holds and we show that each of $[R]$ and $[M]$ is then equivalent to the representation

$$
\left[\mathrm{AV}^{\star}\right] \quad v \oplus w=c \sqrt{\left(\frac{v}{c}\right)^{2}+\left(\frac{w}{c}\right)^{2}-\left(\frac{w}{c}\right)^{2}\left(\frac{v}{c}\right)^{2}},
$$

with $v, w \in[0, c[$, which appears in the case of perpendicular motions [see e.g. 10, Eq. (8)]. We prove, thus, that

$$
[\mathrm{LF}] \Longrightarrow\left([\mathrm{R}] \Longleftrightarrow\left[\mathrm{AV}^{\star}\right] \Longleftrightarrow[\mathrm{M}]\right)
$$

All the results are established under natural regularity and other background conditions on the function $L$ (see Convention 5).

Some recent papers dealing with the axiomatization of special relativity concepts are Andréka et al. [2-4] and Moriconi [9]. In the first three papers, the axiomatization is based on a logical analysis, while in the last one, it is grounded in physical principles. The motivation of the present paper is quite different. As mentioned in our introductory paragraph, our aim was to show 
by an example how functional equations arguments can be used in deriving the consequences of an abstract axiomatization. In passing, however, we came to some results which may be found puzzling, namely implications (2) and (3).

\section{Three preparatory lemmas}

We write $\mathbb{R}_{+}$for the set of (strictly) positive real numbers. The following result is well-known [see 1, p. 79].

Lemma 1. The set of solutions $m:[0,1[\rightarrow \mathbb{R}$ of the functional equation

$$
m\left(\frac{x+y}{1+x y}\right)=m(x) m(y) \quad(x, y \in[0,1[),
$$

with $m$ strictly decreasing, is defined by the equation

$$
m(x)=\left(\frac{1-x}{1+x}\right)^{\xi} \quad(x \in[0,1[),
$$

where $\xi \in \mathbb{R}_{+}$.

Lemma 2. Let $\oplus$ be a real operation on an open interval $\left[0, c\left[\right.\right.$, with $c \in \mathbb{R}_{+}$ and having 0 as its identity element. Suppose that there is some continuous strictly decreasing function $f$ mapping $[0, c[$ onto $] 0,1]$ such that

$$
f(v \oplus w)=f(v) f(w) \quad(v, w \in[0, c[) .
$$

Then there exists a continuous strictly increasing function $u$ mapping $[0, c[$ onto $[0, c[$ and a constant $\xi>0$ such that

$$
f(v)=\left(\frac{c-u(v)}{c+u(v)}\right)^{\xi} \quad(v \in[0, c[) .
$$

Proof. Equation 6 implies that the function $f$ is an isomorphism of $\oplus$ onto the restriction of the multiplicative group $\left(\mathbb{R}_{+}, \cdot\right)$ of the positive reals to the interval $] 0,1]$. But $\left(\mathbb{R}_{+}, \cdot\right)$ is isomorphic to the group $G=(]-1,1[, \odot)$, with the operation $\odot$ defined by

$$
x \odot y=\frac{x+y}{1+x y} .
$$

Thus, $\oplus$ is isomorphic to the restriction of $G$ to the interval [0,1[. Accordingly, there exists a strictly increasing continuous bijection $g:[0,1[\rightarrow[0, c[$ such that

$$
g\left(\frac{x+y}{1+x y}\right)=g(x) \oplus g(y) \quad(x, y \in[0,1[) .
$$


Applying the function $f$ on both sides, we get

$$
\begin{aligned}
(f \circ g)\left(\frac{x+y}{1+x y}\right) & =f(g(x) \oplus g(y)) \\
& =f(g(x)) f(g(y)) \quad(\text { by }(6)),
\end{aligned}
$$

or with $m(x)=(f \circ g)(x)$ for $x \in[0,1[$,

$$
m\left(\frac{x+y}{1+x y}\right)=m(x) m(y) \quad(x, y \in[0,1[) .
$$

Using Lemma 1 , we obtain, with $x \in[0,1[$,

$$
(f \circ g)(x)=m(x)=\left(\frac{1-x}{1+x}\right)^{\xi}=\left(\frac{c-c x}{c+c x}\right)^{\xi} .
$$

For any $x \in[0,1[$, we have $g(x)=v$ for some $v \in[0, c[$. We now define $u(v)=c g^{-1}(v)$. As $g^{-1}:[0,1[\rightarrow[0,1[$ is a continuous strictly increasing bijection, the function $u:[0, c[\rightarrow[0, c[$ is a continuous strictly increasing bijection. From 8, we can thus derive

$$
f(v)=\left(\frac{c-u(v)}{c+u(v)}\right)^{\xi} \quad(v \in[0, c[),
$$

which establishes the lemma.

Definition 3. A function $H: \mathbb{R}_{+} \times\left[0, c\left[\rightarrow \mathbb{R}_{+}\right.\right.$is left order-invariant with respect to similarity transformations if for any $x, z \in \mathbb{R}_{+}, a>0$, and $y, w \in[0, c[$, we have

$$
\text { [LOI] } \quad H(x, y) \leq H(z, w) \Longleftrightarrow H(a x, y) \leq H(a z, w) .
$$

In the sequel, we simply say then that $H$ satisfies [LOI]. The function $H$ is said to satisfy the double cancellation condition if, for all $x, z, t \in \mathbb{R}_{+}$and $y, w, s \in[0, c[$ we have

$$
\text { [DC] } H(x, y) \leq H(z, w) \& H(z, s) \leq H(t, y) \Longrightarrow H(x, s) \leq H(t, w) .
$$

The double cancellation condition appears in ([8], and related references); it is satisfied, for instance, when $H$ stands for the usual addition of real numbers.

Lemma 4. Suppose that a function $L: \mathbb{R}_{+} \times\left[0, c\left[\stackrel{\text { onto }}{\longrightarrow} \mathbb{R}_{+}\right.\right.$is strictly increasing in the first variable, strictly decreasing in the second variable, and continuous in both.

(i) L satisfies Condition [LOI] if and only if there exist two functions $F$ : $\mathbb{R}_{+} \rightarrow \mathbb{R}_{+}$and $f:[0, c[\stackrel{\text { onto }}{\longrightarrow}] 0,1]$, respectively strictly increasing and strictly decreasing, such that $f(0)=1$ and

$$
L(\lambda, v)=F(\lambda f(v)) .
$$


(ii) If $L$ satisfies $[\mathrm{LOI}]$ and, moreover, $L(\lambda, 0)=\lambda$ for all $\lambda \in[0, \infty[$, there exists a continuous function $f$ such that

$$
L(\lambda, v)=\lambda f(v) .
$$

These results are byproducts of Theorems 21 and 38 in [6]. We include a proof for completeness.

Proof. (i) First notice that [LOI] is satisfied when there exist functions $F$ and $f$ as in the statement. Next, suppose that a function $L$ satisfies the stated background conditions and [LOI]. For any constant $a \in \mathbb{R}_{+}$, define the function $\phi_{a}: \mathbb{R}_{+} \rightarrow \mathbb{R}_{+}$by the equation

$$
\left(\phi_{a} \circ L\right)(\lambda, v)=L(a \lambda, v) \quad\left(\lambda \in \mathbb{R}_{+}, v \in[0, c[) .\right.
$$

The $[\mathrm{LOI}]$ condition implies that the function $\phi_{a}$ is well defined and increasing for any $a \in \mathbb{R}_{+}$. It is easily verified that any two functions $\phi_{a}$ and $\phi_{b}$ commute in the sense that $\phi_{a} \circ \phi_{b} \circ L=\phi_{b} \circ \phi_{a} \circ L$. We begin by showing that, under the hypotheses of the lemma, the function $L$ must satisfy the double cancellation condition [DC] introduced in Definition 3. Suppose that, for some $\lambda, \zeta$ and $\theta$ in $\mathbb{R}_{+}$and $v, w$ and $s$ in $[0, c[$, we have

$$
L(\lambda, v) \leq L(\zeta, w) \& L(\zeta, s) \leq L(\theta, v) .
$$

Define $a=\frac{\theta}{\zeta}$ and $b=\frac{\lambda}{\theta}$. We have successively

$$
\begin{aligned}
L(\theta, w)=L(a \zeta, w) & =\left(\phi_{a} \circ L\right)(\zeta, w) \\
& \geq\left(\phi_{a} \circ L\right)(\lambda, v) \\
& =\left(\phi_{a} \circ L\right)(b \theta, v) \\
& =\left(\phi_{a} \circ \phi_{b} \circ L\right)(\theta, v) \\
& \geq\left(\phi_{a} \circ \phi_{b} \circ L\right)(\zeta, s) \\
& =\left(\phi_{b} \circ \phi_{a} \circ L\right)(\zeta, s) \\
& =\left(\phi_{b} \circ L\right)(a \zeta, s) \\
& =\left(\phi_{b} \circ L\right)(\theta, s) \\
& =L(b \theta, s) \\
& =L(\lambda, s) .
\end{aligned}
$$

Thus, (11) implies $L(\theta, w) \geq L(\lambda, s)$. So, the function $L$ satisfies [DC]. By a standard result of measurement theory [see 8, Theorem 2, p. 257] recast in the context of real variables, and the continuity and strict monotonicity assumptions on the function $L$, this implies that there exists three functions $k: \mathbb{R}_{+} \rightarrow \mathbb{R}_{+}, h:\left[0, c\left[\rightarrow \mathbb{R}_{+}\right.\right.$and $G:[0, \infty[\rightarrow[0, \infty[$, with $k$ and $G$ strictly increasing and $h$ strictly decreasing, satisfying

$$
L(\lambda, v)=G(k(\lambda) h(v)) .
$$


From (12), we infer that, for $a, \lambda \in \mathbb{R}_{+}$and $v \in[0, c[$, we have

$$
\left(\phi_{a} \circ G\right)(k(\lambda) h(v))=G(k(a \lambda) h(v)) .
$$

We now rewrite the function $\phi_{a}$ in terms of $k, G$ and $a$. Redefining the function $k$ if need be, we can assume that

$$
h(0)=1 .
$$

Thus, with $v=0,(13)$ becomes

$$
\left(\phi_{a} \circ G\right)(k(\lambda))=G(k(a \lambda)) .
$$

Setting $t=(G \circ k)(\lambda)$, we get, with $\lambda=\left(k^{-1} \circ G^{-1}\right)(t)$

$$
\phi_{a}(t)=\left(G \circ k \circ a k^{-1} \circ G^{-1}\right)(t),
$$

which defines the function $\phi_{a}$ in terms of the functions $G, k$ and the constant $a$. Substituting $\phi_{a}$ in (13) by its expression given by (10), we obtain

$$
\left(G \circ k \circ a k^{-1} \circ G^{-1} \circ G\right)(k(\lambda) h(v))=G(k(a \lambda) h(v)) .
$$

Applying the function $G^{-1}$ on both sides and simplifying, we get

$$
\left(k \circ a k^{-1}\right)(k(\lambda) h(v))=k(a \lambda) h(v) .
$$

Defining now $x=k(\lambda)$ and $y=h(v)$ and $g_{a}=k \circ a k^{-1}$, Eq. 16 can be rewritten as $g_{a}(x y)=g_{a}(x) y$. So, the function $g_{a}$ is necessarily of the form $g_{a}(x)=C(a) x$ for some function $C: \mathbb{R}_{+} \rightarrow \mathbb{R}_{+}$. We have thus $\left(k \circ a k^{-1}\right)(x)=C(a) x$, that is

$$
k(a \lambda)=C(a) k(\lambda),
$$

a Pexider equation defined for all $a$ and $\lambda$ in $\mathbb{R}_{+}$. Because the function $k$ is strictly increasing, the set of solutions of (17) is given by the equations:

$$
\begin{aligned}
& k(\lambda)=A \lambda^{B} \\
& C(a)=a^{B},
\end{aligned}
$$

with positive constants $A$ and $B$ [cf. 1, Theorem 4, p. 144]. Thus, we obtain

$$
L(\lambda, v)=G\left(A \lambda^{B} h(v)\right) \quad\left(\lambda \in \mathbb{R}_{+}, v \in[0, c[) .\right.
$$

Defining the functions

$$
\begin{aligned}
& f(v)=h(v)^{\frac{1}{B}} \quad(x \in[0,1[) \\
& F(t)=G\left(A t^{B}\right) \quad(t \in[0, \infty[),
\end{aligned}
$$

we obtain

$$
\begin{aligned}
L(\lambda, v) & =G\left(A \lambda^{B} h(v)\right)=G\left(A\left(\lambda h(v)^{\frac{1}{B}}\right)^{B}\right) \\
& =F(\lambda f(v)) .
\end{aligned}
$$


Finally, by (14), we have $f(0)=h(0)^{\frac{1}{B}}=1$. (We recall that the function $k$ of (12) may have been redefined to ensure that $h(0)=1$. This redefinition of $k$ is thus affecting the constant $A$ of (18), which is now absorbed by the function $F$.) Thus, $f$ maps $[0,1[$ onto $] 0,1[$. This proves (i).

(ii) This results immediately from (23) and the fact that $f(0)=1$, because

$$
\lambda=L(\lambda, 0)=F(\lambda f(0))=F(\lambda),
$$

and so $F$ is the identity function. Since $L$ is continuous in its second variable, the function $f$ in $(9)$ is continuous.

\section{Main results}

Convention 5. In the sequel, when we write that some function $L: \mathbb{R}_{+} \times$ $\left[0, c\left[\stackrel{\text { onto }}{\longrightarrow} \mathbb{R}_{+}\right.\right.$satisfies the basic hypotheses, we mean that $L$ is strictly increasing in the first variable, strictly decreasing in the second variable, continuous in both, and satisfies [LOI] plus the additional conditions stated in (i) and (ii) in Lemma 4(ii). Accordingly, there exists, by that lemma, a continuous strictly decreasing function $f:[0, c[\stackrel{\text { onto }}{\longrightarrow}] 0,1]$ such that

$$
L(\lambda, v)=\lambda f(v) .
$$

For convenience of reference, we reproduce the formulas of our two axioms. For $\lambda, \lambda^{\prime} \in \mathbb{R}_{+}$and $v, v^{\prime}, w \in[0, c[$ :

$$
\begin{aligned}
& {[\mathrm{R}] \quad L(L(\lambda, v), w)=L(\lambda, v \oplus w) ;} \\
& {[\mathrm{M}] \quad L(\lambda, v) \leq L\left(\lambda^{\prime}, v^{\prime}\right) \Longleftrightarrow L(\lambda, v \oplus w) \leq L\left(\lambda^{\prime}, v^{\prime} \oplus w\right) .}
\end{aligned}
$$

The theorem below concerns the representations of the function $L$ and the operation $\oplus$ in terms of a continuous, strictly increasing function $u:[0, c[\stackrel{\text { onto }}{\longrightarrow}$ $[0, c[$ and a positive constant $\xi$. We also recall the two representations for $v, w \in[0, c[:$

$\left[\mathrm{DE}^{\dagger}\right] \quad L(\lambda, v)=\lambda\left(\frac{c-u(v)}{c+u(v)}\right)^{\xi}$

$\left[\mathrm{AV}^{\dagger}\right] \quad v \oplus w=u^{-1}\left(\frac{u(v)+u(w)}{1+\frac{u(v) u(w)}{c^{2}}}\right)$.

Theorem 6. Suppose that a function $L: \mathbb{R}_{+} \times\left[0, c\left[\stackrel{\text { onto }}{\longrightarrow} \mathbb{R}_{+}\right.\right.$satisfies the basic hypotheses, and let

$$
\oplus:[0, c[\times[0, c[\rightarrow[0, c[\quad(c>0)
$$

be an operation having 0 as its identity element. We assume that $\oplus$ is continuous and strictly increasing in both variables. Under those hypotheses, the following equivalences hold: 


$$
[\mathrm{R}] \Longleftrightarrow\left(\left[\mathrm{DE}^{\dagger}\right] \&\left[\mathrm{AV}^{\dagger}\right]\right) \Longleftrightarrow[\mathrm{M}]
$$

Moreover, the same function $u$ is involved in both $\left[\mathrm{DE}^{\dagger}\right]$ and $\left[\mathrm{AV}^{\dagger}\right]$.

Proof. We successively prove: (i) $[\mathrm{R}] \Rightarrow\left[\mathrm{DE}^{\dagger}\right]$; (ii) $[\mathrm{R}] \Rightarrow\left[\mathrm{AV}^{\dagger}\right]$; (iii) ([DE $\left.{ }^{\dagger}\right] \&$ $\left.\left[\mathrm{AV}^{\dagger}\right]\right) \Rightarrow[\mathrm{M}]$; (iv) $[\mathrm{M}] \Rightarrow[\mathrm{R}]$.

(i) $[\mathrm{R}] \Rightarrow\left[\mathrm{DE}^{\dagger}\right]$. By Lemma 4 and Axiom $[\mathrm{R}]$, we get

$$
\begin{aligned}
\lambda f(v \oplus w) & =L(\lambda, v \oplus w)=L(L(\lambda, v), w)=L(\lambda, v) f(w) \\
& =\lambda f(v) f(w),
\end{aligned}
$$

and so, with $f:[0, c[\stackrel{\text { onto }}{\longrightarrow}] 0,1]$ strictly decreasing and continuous,

$$
f(v \oplus w)=f(v) f(w) \quad(v, w \in[0, c[) .
$$

Applying Lemma 2, we get

$$
f(v)=\left(\frac{c-u(v)}{c+u(v)}\right)^{\xi}
$$

for some constant $\xi>0$ and some continuous, strictly increasing function $u:\left[0, c\left[\stackrel{\text { onto }}{\longrightarrow}\left[0, c\left[\right.\right.\right.\right.$. So, as $L(\lambda, v)=\lambda f(v),\left[\mathrm{DE}^{\dagger}\right]$ holds.

(ii) $[\mathrm{R}] \Rightarrow\left[\mathrm{AV}^{\dagger}\right]$. From $(26)$, we get for $\left.\left.z \in\right] 0,1\right]$

$$
f^{-1}(z)=u^{-1}\left(c \times \frac{1-z^{1 / \xi}}{1+z^{1 / \xi}}\right) .
$$

Using now (25-27), we obtain

$$
\begin{aligned}
v \oplus w & =f^{-1}(f(v) f(w)) \\
& =f^{-1}\left(\left(\frac{c-u(v)}{c+u(v)}\right)^{\xi}\left(\frac{c-u(w)}{c+u(w)}\right)^{\xi}\right) \\
& =u^{-1}\left(c \times \frac{1-\left(\frac{c-u(v)}{c+u(v)}\right)\left(\frac{c-u(w)}{c+u(w)}\right)}{1+\left(\frac{c-u(v)}{c+u(v)}\right)\left(\frac{c-u(w)}{c+u(w)}\right)}\right) \\
& =u^{-1}\left(\frac{u(v)+u(w)}{1+\frac{u(v) u(w)}{c^{2}}}\right),
\end{aligned}
$$

after simplifications. We have thus proved the implication $[\mathrm{R}] \Rightarrow\left(\left[\mathrm{DE}^{\dagger}\right] \&\right.$ $\left.\left[\mathrm{AV}^{\dagger}\right]\right)$. tion

(iii) $\left(\left[\mathrm{DE}^{\dagger}\right] \&\left[\mathrm{AV}^{\dagger}\right]\right) \Rightarrow[\mathrm{M}]$. In the derivation below, we use the abbrevia-

$$
f(v)=\left(\frac{c-u(v)}{c+u(v)}\right)^{\xi} .
$$

By $\left[\mathrm{AV}^{\dagger}\right]$ and calculations similar to $(28-30)$ we derive that

$$
f(v \oplus w)=f(v) f(w) .
$$


For any $\lambda, \lambda^{\prime} \in \mathbb{R}_{+}$and $v, v^{\prime}, w \in[0, c[$, we now have

$$
\begin{aligned}
L(\lambda, v) & \leq L\left(\lambda^{\prime}, v^{\prime}\right) & & \\
& \Longleftrightarrow \lambda f(v) \leq \lambda^{\prime} f\left(v^{\prime}\right) & & \text { (by } \left.\left[\mathrm{DE}^{\dagger}\right]\right) \\
& \Longleftrightarrow \lambda f(v) f(w) \leq \lambda^{\prime} f\left(v^{\prime}\right) f(w) & & (\text { since } f(w)>0) \\
& \Longleftrightarrow \lambda f(v \oplus w) \leq \lambda^{\prime} f\left(v^{\prime} \oplus w\right) & & \text { (by }(31)) \\
& \Longleftrightarrow L(\lambda, v \oplus w) \leq L\left(\lambda^{\prime}, v \oplus w\right) & & \text { (by } \left.\left[\mathrm{DE}^{\dagger}\right]\right),
\end{aligned}
$$

and so $[\mathrm{M}]$ holds.

(iv) $[\mathrm{M}] \Rightarrow[\mathrm{R}]$. An immediate consequence of $[\mathrm{M}]$ is that there exists, for any $w \in\left[0, c\left[\right.\right.$, a function $K_{w}: \mathbb{R}_{+} \rightarrow \mathbb{R}_{+}$such that

$$
K_{w}(L(\lambda, v))=L(\lambda, v \oplus w) \quad\left(\lambda \in \mathbb{R}_{+}, v \in[0, c[) .\right.
$$

(Axiom $[\mathrm{M}]$ ensures that $K_{w}$ is well defined by the above equation.) Applying the basic hypotheses and Lemma 4, Eq. 32 becomes

$$
K_{w}(\lambda f(v))=\lambda f(v \oplus w) .
$$

Setting $v=0$, we obtain $0 \oplus w=w$ in the r.h.s. of (33), and since $f(0)=1$ by Lemma 4, we get

$$
K_{w}(\lambda)=\lambda f(w)
$$

This allows us to rewrite $(33)$ as $\lambda f(v) f(w)=\lambda f(v \oplus w)$, or equivalently, by $(24)$,

$$
L(L(\lambda, v), w)=L(\lambda, v \oplus w) .
$$

Thus, $[\mathrm{R}]$ holds. This completes the proof of the theorem.

In the next corollary, we assume that the operation $\oplus$ satisfies the standard formula for the relativistic addition of velocities:

$$
[\mathrm{AV}] \quad v \oplus w=\frac{v+w}{1+\frac{v w}{c^{2}}} \quad(v, w \in[0, c[) .
$$

As a result, either $[\mathrm{M}]$ or $[\mathrm{R}]$ imply that the function $L$ has a form which only differs from the Doppler operator by a unspecified positive exponent $\xi$.

Corollary 7. Suppose that the basic hypotheses are satisfied by a function $L$ : $\mathbb{R}_{+} \times\left[0, c\left[\stackrel{\text { onto }}{\longrightarrow} \mathbb{R}_{+}\right.\right.$, and let $\oplus$ be defined by the standard formula $[\mathrm{AV}]$ for the relativistic addition of velocities. Then the following three equivalences hold:

$$
[\mathrm{M}] \Longleftrightarrow\left[\mathrm{DE}^{\star}\right] \Longleftrightarrow[R]
$$

with, for some exponent $\xi \in \mathbb{R}_{+}$,

$$
\left[\mathrm{DE}^{\star}\right] \quad L(\lambda, v)=\lambda\left(\frac{1-\frac{v}{c}}{1+\frac{v}{c}}\right)^{\xi} \quad(v, w \in[0, c[) .
$$


Proof. As $\left[\mathrm{DE}^{\star}\right]$ is a special case of $\left[\mathrm{DE}^{\dagger}\right]$ and $[\mathrm{AV}]$ implies $\left[\mathrm{AV}^{\dagger}\right]$, we know by Theorem 6 that $\left[\mathrm{DE}^{\star}\right] \Rightarrow[\mathrm{M}] \Leftrightarrow[\mathrm{R}]$. It suffices, thus, to establish that $[\mathrm{M}]$ $\Rightarrow\left[\mathrm{DE}^{\star}\right]$. We only sketch the argument (which is familiar from our proof of Theorem 6).

Axiom $[\mathrm{M}]$ implies that

$$
K_{w}(L(\lambda, v))=L(\lambda, v \oplus w) \quad\left(\lambda \in \mathbb{R}_{+}, v \in[0, c[)\right.
$$

for some function $K_{w}$. By Lemma 4, we get $K_{w}(\lambda f(v))=\lambda f(v \oplus w)$. Setting $v=0$ yields $K_{w}(\lambda)=\lambda f(w)$. We can thus rewrite (34), after cancelling the $\lambda$ 's, as

$$
f(v) f(w)=f\left(\frac{v+w}{1+\frac{v w}{c^{2}}}\right) .
$$

Defining $m:[0,1[\rightarrow[0,1[: x \mapsto m(x)=f(c x)$, we obtain

$$
m(x) m(y)=m\left(\frac{x+y}{1+x y}\right) \quad(x, y \in[0,1[),
$$

with $m$ strictly decreasing and continuous. From Lemma 1, we get

$$
f(c x)=m(x)=\left(\frac{1-x}{1+x}\right)^{\xi}
$$

with $\xi>0$. With $v=c x$, Lemma 4 and (37) give

$$
L(\lambda, v)=\lambda f(v)=\lambda\left(\frac{1-\frac{v}{c}}{1+\frac{v}{c}}\right)^{\xi} \quad(v \in[0, c[) .
$$

Thus, $\left[\mathrm{DE}^{\star}\right]$ holds.

Remarks 8. (a) Corollary 7 tells us that if the operator $\oplus$ is defined by $[\mathrm{AV}]$, then Axioms $[\mathrm{R}]$ and $[\mathrm{M}]$ are equivalent to [DE*]. However, whatever the value of its exponent $\xi>0$, Formula [DE*] is inconsistent with the Lorentz-Fitzgerald Contraction

$$
\text { [LF] } \quad L(\lambda, v)=\lambda \sqrt{1-\left(\frac{v}{c}\right)^{2}} .
$$

The point is that $[\mathrm{LF}]$ and $\left[\mathrm{DE}^{*}\right]$ represent essentially different functions for any fixed positive value of the exponent $\xi$ in [DE*]. Thus, if the standard formula for the relativistic addition of velocities is assumed, then the Lorentz-Fitzgerald Contraction is inconsistent with the two axioms $[R]$ and $[M]$, a fact which may be worth pondering (see our discussion in the last section).

(b) On the other hand, if we do not assume a priori that the operation $\oplus$ is defined by the standard formula $[\mathrm{AV}]$, then the Generalized Doppler 
Formula $\left[\mathrm{DE}^{\dagger}\right]$ is consistent with the Lorentz-Fitzgerald operator in the sense that, setting $\xi=\frac{1}{2}$, the equation

$$
\sqrt{\frac{c-u(v)}{c+u(v)}}=\sqrt{1-\left(\frac{v}{c}\right)^{2}}
$$

can be solved for the function $u$. The solution is

$$
u(v)=\frac{c\left(\frac{v}{c}\right)^{2}}{2-\left(\frac{v}{c}\right)^{2}} .
$$

This raises the question: what is the form of an operation $\oplus$ that is consistent with $[\mathrm{R}],[\mathrm{M}]$ and the Lorentz-Fitzgerald Contraction? Our next corollary answers the question.

Corollary 9. Let $L: \mathbb{R}_{+} \times\left[0, c\left[\stackrel{\text { onto }}{\longrightarrow} \mathbb{R}_{+}\right.\right.$be a function satisfying the basic hypotheses and suppose that

$$
\oplus:[0, c[\times[0, c[\rightarrow[0, c[\quad(c>0)
$$

is an operation that is continuous and strictly increasing in both variables and has 0 as its identity element. If the function L satisfies the Lorentz-Fitzgerald Contraction [LF], then the following three equivalences hold:

$$
[\mathrm{M}] \Longleftrightarrow\left[\mathrm{AV}^{\star}\right] \Longleftrightarrow[\mathrm{R}]
$$

with, for $v, w \in[0, c[$,

$$
\left[\mathrm{AV}^{\star}\right] \quad v \oplus w=c \sqrt{\left(\frac{v}{c}\right)^{2}+\left(\frac{w}{c}\right)^{2}-\left(\frac{w}{c}\right)^{2}\left(\frac{v}{c}\right)^{2}} .
$$

Note that, for $v, w \in\left[0, c\left[,\left[\mathrm{AV}^{\star}\right]\right.\right.$ can be rewritten as

$$
\begin{aligned}
v \oplus w & =c \sqrt{\left(\frac{v}{c}\right)^{2}\left(1-\left(\frac{w}{c}\right)^{2}\right)+\left(\frac{w}{c}\right)^{2}} \\
& =c \sqrt{\left(\frac{w}{c}\right)^{2}\left(1-\left(\frac{v}{c}\right)^{2}\right)+\left(\frac{v}{c}\right)^{2}},
\end{aligned}
$$

so that $\oplus$ is strictly increasing in both variables and also satisfies

$$
\begin{gathered}
0 \leq v \oplus w<c \\
\lim _{v \rightarrow c} v \oplus w=\lim _{w \rightarrow c} v \oplus w=c,
\end{gathered}
$$

and

$$
0 \oplus v=v \oplus 0=v
$$


Proof. We first show that $[\mathrm{LF}]$ and $\left[\mathrm{AV}^{\star}\right]$ are special cases of $\left[\mathrm{DE}^{\dagger}\right]$ and $\left[\mathrm{AV}^{\dagger}\right]$, respectively. Beginning with $[\mathrm{LF}]$, we have by $\left[\mathrm{DE}^{\dagger}\right]$, with $\xi=\frac{1}{2}$,

$$
L(\lambda, v)=\lambda \sqrt{\frac{c-u(v)}{c+u(v)}} \quad\left(\lambda \in \mathbb{R}_{+}, v \in[0, c[) .\right.
$$

Replacing $u(v)$ by its expression in (39) gives [LF].

We prove that $\left[\mathrm{AV}^{\star}\right]$ is a special case of $\left[\mathrm{AV}^{\dagger}\right]$ in the same way, that is, by replacing the function $u$ in $\left[\mathrm{DE}^{\dagger}\right]$ by its expression in (39) - thus

$$
u^{-1}(t)=c \sqrt{\frac{2 t}{c+t}} .
$$

After some straightforward manipulations, which we omit, we obtain the representation:

$$
\begin{aligned}
v \oplus w & =u^{-1}\left(\frac{u(v)+u(w)}{1+\frac{u(v) u(w)}{c^{2}}}\right) \\
& =c \sqrt{\left(\frac{v}{c}\right)^{2}+\left(\frac{w}{c}\right)^{2}-\left(\frac{w}{c}\right)^{2}\left(\frac{v}{c}\right)^{2}} .
\end{aligned}
$$

By Theorem 6 , we have $[\mathrm{R}] \Leftrightarrow\left(\left[\mathrm{DE}^{\dagger}\right] \&\left[\mathrm{AV}^{\dagger}\right]\right) \Leftrightarrow[\mathrm{M}]$. So far, we have thus established that

$$
[\mathrm{LF}] \Longrightarrow\left(\left[\mathrm{AV}^{\star}\right] \Rightarrow[\mathrm{R}] \&[\mathrm{M}]\right) .
$$

It suffices, thus, to show that the following holds:

$$
[\mathrm{LF}] \Rightarrow\left([\mathrm{R}] \Rightarrow\left[\mathrm{AV}^{\star}\right]\right) .
$$

Suppose that both $[\mathrm{LF}]$ and $[\mathrm{R}]$ are true. We have thus

$$
\begin{aligned}
L(L(\lambda, v), w) & =\lambda \sqrt{1-\left(\frac{v}{c}\right)^{2}} \sqrt{1-\left(\frac{w}{c}\right)^{2}} \\
& =\lambda \sqrt{1-\left(\frac{v \oplus w}{c}\right)^{2}},
\end{aligned}
$$

which implies

$$
\left(1-\left(\frac{v}{c}\right)^{2}\right)\left(1-\left(\frac{w}{c}\right)^{2}\right)=1-\left(\frac{v \oplus w}{c}\right)^{2} .
$$

Solving for $v \oplus w$ yields $\left[\mathrm{AV}^{\star}\right]$. 


\section{Summary and discussion}

The objective of this paper was to show how functional equations arguments could be used to leverage the consequences of abstract axiomatization of empirical phenomena, thereby giving possibly new insights into the basic structure of such phenomena. We chose the relativistic Doppler effect [DE] and the relativistic addition of velocities $[\mathrm{AV}]$ as our example, with the two basic axioms $[R]$ and $[M]$. We proved in Theorem 6 that each of the two axioms was equivalent to the following generalizations of $[\mathrm{DE}]$ and $[\mathrm{AV}]$, taken jointly: for some function $u$ and some positive parameter $\xi$,

$\left[\mathrm{DE}^{\dagger}\right] \quad L(\lambda, v)=\lambda\left(\frac{c-u(v)}{c+u(v)}\right)^{\xi}$,

$\left[\mathrm{AV}^{\dagger}\right] \quad v \oplus w=u^{-1}\left(\frac{u(v)+u(w)}{1+\frac{u(v) u(w)}{c^{2}}}\right)$.

Thus, $[\mathrm{DE}]$ and $[\mathrm{AV}]$ are obtained when the function $u$ is the identity and $\xi=\frac{1}{2}$. So, the two axioms $[\mathrm{R}]$ and $[\mathrm{M}]$ appear to be consistent with the standard representation of the phenomena.

However, the picture gets more complicated when we look more closely at the results, specifically Corollaries 7 and 9 of Theorem 6 .

Corollary 7 is summarized by the implication

$$
[\mathrm{AV}] \Longrightarrow\left([\mathrm{R}] \Longleftrightarrow\left[\mathrm{DE}^{\star}\right] \Longleftrightarrow[\mathrm{M}]\right),
$$

with

$\left[\mathrm{DE}^{\star}\right] \quad L(\lambda, v)=\lambda\left(\frac{1-\frac{v}{c}}{1+\frac{v}{c}}\right)^{\xi}$.

This implies that if the standard formula $[\mathrm{AV}]$ for the addition of velocities holds, then the two axioms $[\mathrm{R}]$ and $[\mathrm{M}]$ are inconsistent with the LorentzFitzgerald Contraction.

Note that, by themselves (that is, without assuming that [AV] holds), these axioms are consistent with $[\mathrm{LF}]$. Indeed, $[\mathrm{R}]$ and $[\mathrm{M}]$ imply $\left[\mathrm{DE}^{\dagger}\right]$ by Theorem 6 and, as pointed out in our Remark 8(b), $\left[\mathrm{DE}^{\dagger}\right]$ becomes $[\mathrm{LF}]$ for $\xi=\frac{1}{2}$ and

$$
u(v)=\frac{c\left(\frac{v}{c}\right)^{2}}{2-\left(\frac{v}{c}\right)^{2}} .
$$

In the framework of Corollary 7, the form of the function $u$ given by Equation 41 must also be used to rewrite $\left[\mathrm{AV}^{\dagger}\right]$, which leads to a different form for the addition of velocities. Rather than computing this form directly, we get it as part of the proof of Corollary 9. This corollary is summarized by implication (3) reproduced below

$$
[\mathrm{LF}] \Longrightarrow\left([\mathrm{R}] \Longleftrightarrow\left[\mathrm{AV}^{\star}\right] \Longleftrightarrow[\mathrm{M}]\right),
$$


with

$$
\left[\mathrm{AV}^{\star}\right] \quad v \oplus w=c \sqrt{\left(\frac{v}{c}\right)^{2}+\left(\frac{w}{c}\right)^{2}-\left(\frac{w}{c}\right)^{2}\left(\frac{v}{c}\right)^{2}} .
$$

So, in the framework of Axioms $[\mathrm{R}]$ and $[\mathrm{M}]$, which are equivalent, we can have either $[\mathrm{LF}]$ or $[\mathrm{AV}]$, but not both. We must conclude that these axioms must violate some basic principle of special relativity. We did not try to elucidate this contradiction, but it is of interest because the axioms are compelling.

Open Access. This article is distributed under the terms of the Creative Commons Attribution Noncommercial License which permits any noncommercial use, distribution, and reproduction in any medium, provided the original author(s) and source are credited.

\section{References}

[1] Aczél, J.: Lectures on Functional Equations and their Applications. Academic Press, New York (1966)

[2] Andréka, H., Madarász, J.X., Németi, I.: Logical axiomatization of space-time. Samples from the literature. In: Prékopa, A., Molnár, E. (eds.) Non-Euclidean Geometries. Mathematics and its Applications, pp. 155-185. Springer, Berlin (2006)

[3] Andréka, H., Madarász, J.X., Székeli, G.: Twin paradox and the logical foundation of relativity theory. Found. Phys. 35(5), 681-714 (2006)

[4] Andréka, H., Madarász, J.X., Székeli, G.: Axiomatizing relativistic dynamics without conservation postulates. Studia Logica 89(2), 163-186 (2008)

[5] Ellis, G.F.R., Williams, R.M.: Flat and Curved Space-Times. Clarendon Press, Oxford (1988)

[6] Falmagne, J.-Cl.: Meaningfulness and order invariance: two fundamental principles for scientific laws. Found. Phys. 9, 1341-1384 (2004)

[7] Feynman, R.P., Leighton, R.B., Sands, M.: The Feynman Lectures on Physics. AddisonWesley, Reading (1963)

[8] Krantz D.H., Luce R.D., Suppes P., Tversky A.: Foundations of Measurement, Volume 1: Additive and Polynomial Representations. Academic Press, New York (1971)

[9] Moriconi, M.: Special theory of relativity through the Doppler effect. Eur. J. Phys. 27, 1409-1423 (2006)

[10] Ungar, A.A.: Thomas precession and its associated grouplike structure. Am. J. Phys. 59(9), 824-834 (1991)

Jean-Claude Falmagne

University of California

Irvine

CA

USA

e-mail: jcf@uci.edu

Jean-Paul Doignon

Université Libre de Bruxelles

Bruxelles

Belgium

e-mail: doignon@ulb.ac.be

Received: February 18, 2010

Revised: June 21, 2010 